\title{
Molecular Imaging Using PET/MRI Particle
}

\author{
Do Won Hwang ${ }^{1,2}$, Hyewon Youn ${ }^{1,2}$ and Dong Soo Lee $e^{* 1,2,3}$ \\ ${ }^{I}$ Department of Nuclear Medicine, College of Medicine, ${ }^{2}$ Institute of Radiation Medicine, Medical Research Center, \\ ${ }^{3}$ Department of Molecular Medicine and Biopharmaceutical Science, WCU Graduate School of Convergence Science \\ and Technology, Seoul National University, Seoul, Korea
}

\begin{abstract}
With the remarkable progress of non-invasive molecular imaging techniques, the nanotechnology could provide powerful tools for development of diagnostic and therapeutic agents in bio-and nano-medicine field. Several imaging techniques including PET and MRI system have been developed for diagnosis, providing functional and anatomical information. Recently, the combined system of PET/MRI imaging modalities with nanoparticles allows the simultaneous monitoring of cancer localization and provides detailed information of nanoparticle distribution in vivo. A variety form of multimodal imaging probes has been developed to overcome the limitation of single imaging modality.

In this review, we describe the technological advances associated with nanoparticle-based multimodal imaging system including PET/MRI in living subjects. First, we introduce the simple imaging strategy using optical or PET imaging tracer and the MR imaging approach using a variety of magnetic nanoparticles for the detection of cancer. Then, we cover the improved techniques for efficient targeting with multimodal imaging agents to lymph node area and engrafted cancer sites. In addition, we introduce a successful application of quadruple imaging nanoparticles for the evaluation of more accurate tumor distribution in clinical oncology. These results indicate that multimodal imaging strategy using nanoparticles can endow broad application in multidisciplinary field to monitor a variety of biological systems in a single investigation, and ultimately offer synergistic information for better understanding in diagnosis and therapy of human diseases.
\end{abstract}

Keywords: PET/MRI imaging, nanoparticle, multimodal imaging, cell tracking.

\section{INTRODUCTION}

Owing to rapid advance of imaging technology, the development of imaging modalities such as positron emission tomography (PET) and magnetic resonance imaging (MRI) has provided us the great opportunities to enlarge our understanding of non-invasive diagnostic application for a wide variety of diseases and abnormalities [1-3]. Each imaging modality has been developed in clinical field, showing its own unique advantage such that PET imaging modality using radioactive isotope enables measurement of metabolic processes with high signal sensitivity and MR imaging modality offers high spatial resolution with great soft tissue contrast. Nevertheless, the major limitation caused by individual imaging modality such as poor resolution for PET and low signal sensitivity for MRI remains to be solved for clinical application. Recently, the combination of PET and MRI systems was introduced to provide multifunctional information for the more precise interpretation of human disease. This multimodal imaging system can provide potential benefits to overcome the defects occurred in each imaging system by offering complementary information to each other [4].

Various forms of multimodal imaging platforms have been exploited such as triple fusion reporter imaging system

*Address correspondence to this author at the Department of Nuclear Medicine, Medical Research Center, Seoul National University College of Medicine, 28 Yongon-dong, Jongno-gu, Seoul, 110-744, Korea; Tel: +82 (2) 2072 2501; Fax: +82 (2) 3668 7090; E-mail: ds1@plaza.snu.ac.kr and small molecules-based multiplex imaging [5-7]. However, multimodal imaging system using small molecules has the limitation that numerous attachment points are not available in their surface for linkage with drug and imaging moieties.

Recently, the development of nanotechnology using nano-sized materials has been pivotal in drug development, nanosurgery and nanoscience for examining a wide range of biological events [8-12]. Based on its potential merits, the application of nanomaterials as delivery carriers is necessary for the cell tracking in vivo imaging. The nanoparticles having a wide range of moieties for various functional groups on their surface are suitable for multimodal imaging system. Many trials of nanotechnology-based molecular imaging for biological application have been attempted to elucidate the protein function, siRNA delivery and thermal therapeutic effect [13-15]. Furthermore, several studies using PET and MRI imaging system suggested that the radiolabeled multifunctional magnetic nanocarriers are effective in evaluating cancer detection and therapy for clinical use [16]. Another interesting approach also demonstrated the effective use of multimodal nanoprobe using hybrid PET/MR imaging system, exhibiting identical imaging signal for PET and MR image at the same spot. Accurate localization of PET/MR imaging probes was found in sentinel lymph node for diagnosis of cancer metastasis [17]. Here we review the current state of the art on the development of multimodal nanoparticle imaging system based on PET and MRI hybrid devices, and discuss about 
possible use of those systems in the cancer detection and biological diagnostics.

\section{NANOPARTICLE-BASED SYSTEMS \\ CELL TRACKING}

The development of cell specific delivery system has been regarded as one of the major goals in nanomedicine field. This point is critically associated with cancer therapy which possesses the challenges to minimize the side effect caused by non-specific accumulation of drug in normal cells. In addition, in order to optimize cancer targeting system using nanoparticle in vivo, efficient imaging agents are required for tracking of cancer localization.

Currently, molecular imaging technology has rapidly developed in non-invasive manner, capable of monitoring numerous biological processes at molecular and cellular levels. The development of efficient imaging probes has accelerated the possibility to detect the grafted cell migration in vivo and evaluate drug response in the molecular imaging field, offering repetitive imaging detection, signal quantification and tomographic capabilities. Extensive researches related to in vivo cancer targeting system using molecular imaging sensors have been intensively studied for development of cancer biomarkers as well as for noninvasive cancer treatment in nanomedicine field. One of the promising cancer biomarkers, $\alpha_{v} \beta_{3}$ integrin-based molecular imaging strategy has been investigated for targeted cancer therapy using RGD peptide-labled nanomaterials. Cai et al. reported the successful use of near-infrared-emitting quantum dots conjugated with RGD ligands for the detection of tumor vasculature [18]. Due to the availability of attachment of numerous RGD peptides on the surface of quantum dots, significant optical signal was specifically detected in the tumor sites. However, the light scattering problem due to penetration in deep tissue is the major limitation, which would be hard to apply the fluorescence imaging probes in human medicine. To overcome this obstacles, a variety sort of RGD-labeled magnetic nanoparticles including ultrasmall superparamagnetic iron oxide (USPIO) for a better MR image quality were applied for clinical use to monitor selective cancer detection $[19,20]$. Also, a wide range of cancer-targeted imaging tools using ultrasound-based microbubble probe or Raman signal-based single-walled carbon nanotubes (SWNT) tracer have been exploited to establish optimal cancer targeting agents for clinical application [21,22]. One remarkable approach relative to cancer targeted imaging system using magnetic nanocarriers revealed specific visualization of magnetic signal in grafted tumor site using fabricated magnetic imaging nanoprobes [23]. The ultra-sensitive MR imaging nanoprobe was selected to yield better MR signal contrast for in vivo detection of cancer cells, by examining MR contrast effects from comparison with magnetismengineered iron oxides (MEIO) doping with different metal ion. The magnetic characteristics of newly developed MnMEIO nanoparticle showing the highest MR signal intensity compared to original MEIO nanoparticle were demonstrated, and the specific targeting of herceptinengineered MnMEIO nanoparticle was clearly visualized in thigh of mice bearing Her2-positive cancer cell lines. Likewise, cancer targeting approach using MR nanoprobes capable of the tomographic analysis can provide promising technologies for early cancer detection and screening. However, based on in vivo targeting systems using magnetic nanoparticles, it is important to note that critical concerns for significant non-specific MR signal that arises from accumulation of nanoparticles in activated macrophage remain to be challenged. In addition, as we mentioned in introduction part, one of the common drawbacks for the use of MR imaging modality is low sensitivity. One study using radionuclide imaging nanoparticle probe represented the specific targeting of ${ }^{64} \mathrm{Cu}$-labeled nanoparticle into pulmonary endothelium area with high sensitivity [24]. However, the limitation incapable of providing anatomical information prevents radionuclide imaging from progressing further to produce better imaging quality.

The non-invasive imaging strategy using MR or PET tracer also enables to track the stem cells in vivo. A wide range of studies using magnetic particle has been progressed in stem cell research in terms of stem cell-based therapy [25$28]$. One comprehensive study described a potential tracking system of stem cells labeled with magnetic particle in brain region [25]. The localization of bone marrow-derived stem cell possessing superparamagnetic (SPIO) particles to the tumor region was visualized in the brain tumor model, showing hypointense MR signal in glioma-implanted site. This in vivo imaging approach based on MR imaging probe for stem cell tracking into cancer cells could provide the valuable information to evaluate the fate of engrafted stem cells and the therapeutic efficacy of stem cells in terms of cancer therapy. Another approach of stem cell tracking using magnetic particle was carried out in myocardial infarction model [28]. Chapon et al. introduced a FDG-PET imaging system for measuring myocardial viability in addition to fundamental tracking system of stem cell using iron oxide (IO)-labeled stem cells, showing the retention of bone marrow stem cell (BMSC) in infracted heart area from MR image up to 6 week and significantly increased FDG uptake in BMSC-implanted heart region. Recent approach using magnetic labeling technique in addition to PET reporter gene was developed as a cell tracking model [29].

\section{DUAL-MODE IMAGING NANOPARTICLE BASED ON PET/MRI}

Although single imaging modality has been used for broad application in a variety of imaging field with faithful implementing its own potential advantages, it is constrained by own limitation that can not offer all the required information, and eventually this challenge brings the appearance of multimodal imaging era for biomedical application. Various forms of hybrid imaging systems that combine two or more of imaging modalities have been developed to provide more precise detection of disease and abnormal function for clinical application. Especially, the studies of PET/MR dual modality have revealed as powerful research tools in terms of anatomical and functional imaging acquisition, simultaneously. Several studies have investigated the usefulness of the combined PET and MRI systems through a single nanoparticle probe in biological subject or tumor model $[16,17,30]$. This hybrid PET/MR modality was also used for sentinel lymph node mapping for the detection of cancer metastasis. Previous investigation for the detection of lymph node was mostly based on the fluorescence imaging alone using quantum dots in 
biomedical imaging field $[31,32]$. Although the development of near-infrared quantum dots are somewhat available in deep tissue, the depth penetration problem has yet been the major limitation. Based on this point, Choi et al. demonstrated that magnetic imaging sensor labeled with PET imaging probe was shown to be effective for the tracking of sentinel lymph nodes [17]. The original form of MnMEIO magnetic particle which shows enhanced MR signal contrast was modified with ${ }^{124} \mathrm{I}$ radioisotope attachment, mediated by serum albumin to improve colloidal stability in broad $\mathrm{pH}$ condition. The overlaid PET and MR fusion image indicated that the matched position of brachial lymph node through PET and MRI image acquisition was significantly detected after administration of ${ }^{124}$ I-labeled MnMEIO nanoparticle into rat forepaw (Fig. 1). However, the retention of the nanoparticles in injection site would be challengeable. This problem would be solved by the injection of lower concentration of the nanoparticles or the additional administration of solvent such as PBS as soon as the nanoparticles were injected into the sole of a foot for tracking lymph node.

This hybrid PET/MRI modality system can reflect synergistic effects over single modality alone for multimodal diagnosis. Another report using the bi-functional imaging probe suggested in vivo tumor targeting system for cancer diagnosis and therapy in clinical oncology part. As mentioned above, cyclic arginine-glycine-aspartic (RGD) peptides which are known as a potential cancer targeting ligand were also applied for the multimodal imaging for cancer detection. Lee et al. reported the RGD-based dualmode modality system for cancer imaging using magnetic iron oxide with the addition of PET imaging radioisotope through DOTA chelating agents [16]. The synthesized ${ }^{64} \mathrm{Cu}-$ DOTA-IO-RGD particle intravenously injected into mouse was specifically targeted to grafted tumor site in time dependent manner, providing comprehensive information of functional and anatomical data, simultaneously. The application of PET/MRI imaging probes for the detection of cancer can lead to potential benefit of combining substantial information from these imaging techniques to improve early diagnostic accuracy in cancer research.

\section{IN VIVO CELLULAR IMAGING USING A TRIPLE OR QUADRUPLE NANOPARTICLE IMAGING PROBE}

As the necessity to elucidate the complicated phenomenon in biological system is being increased, the emergence of a new class of multiple probes equipped with more than three imaging modality is required. From this point, the application of nanomatrials providing a large number of functional attachment points in their surface is suitable for establishing triple or quadruple imaging modality systems. One remarkable research revealed successful visualization of vulnerable plaque legions using trimodality imaging probes in mice [33]. The authors represented not only specific radioactivity of ${ }^{64} \mathrm{Cu}-\mathrm{TNP}$ (trireporter nanoparticle) in atherosclerotic plaque region of aortas and carotid arteries, by tracking macrophage activity in $a p o E^{-/-}$mice, but also the detailed information of cellular distribution through fluorescent properties ex vivo (Fig. 2). This hybrid-type of triple imaging nanoprobe capable of simultaneous monitoring of PET, MR and fluorescent imaging can offer versatile capability for accurate validation of localization of target site in a single investigation. Recently, our group developed quadruple imaging modality
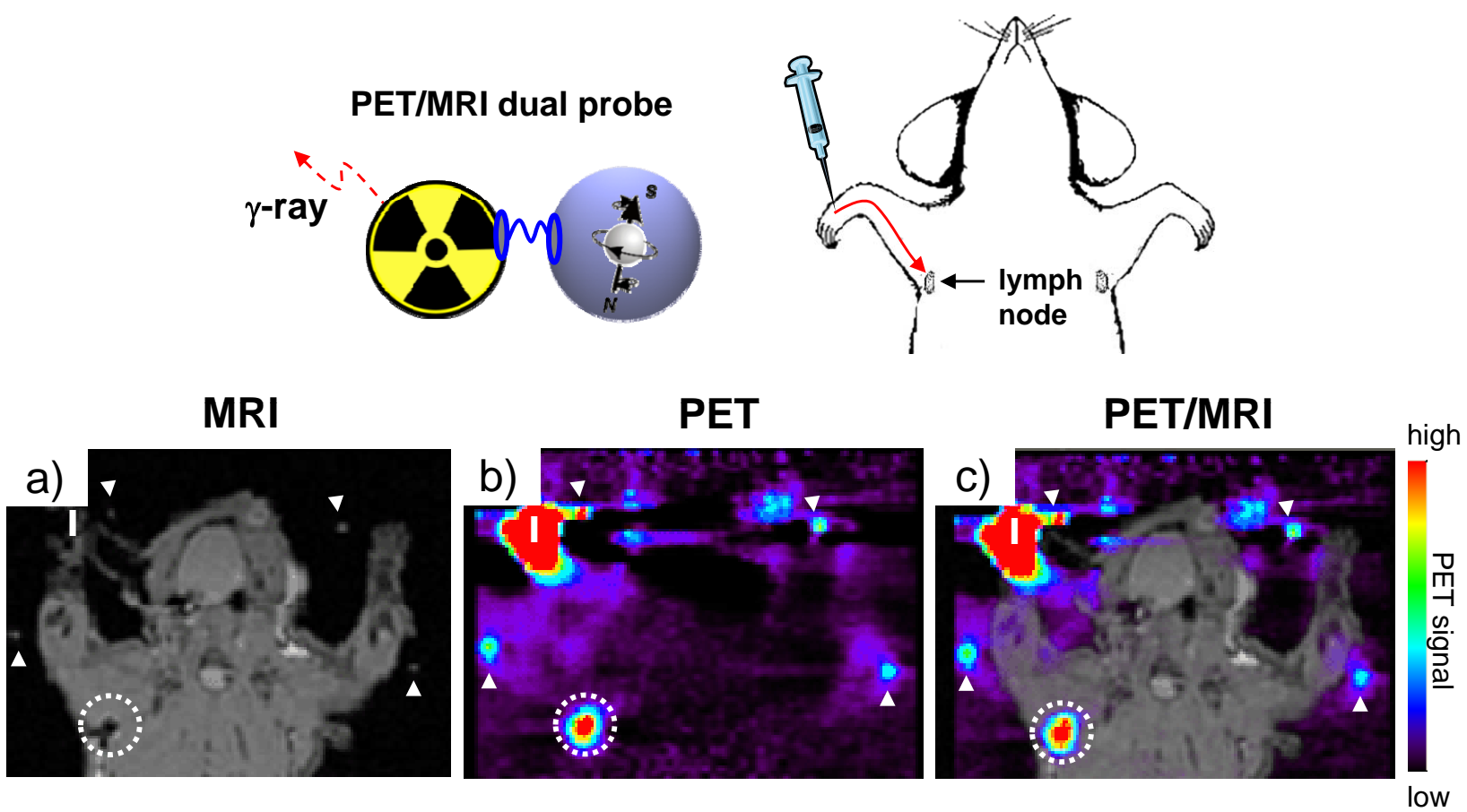

Fig. (1). Dual-mode imaging for targeting sentinel lymph node using PET/MR nanoprobes. ${ }^{124}$ I labeled SA-MnMEIO through surface modification using serum albumin (SA) was designed to establish Hybrid PET/MR nanosensor. An hour after ${ }^{124}$ I-SA-MnMEIO nanoparticle was administered into right forepaw, specific targeting of ${ }^{124}$ I-SA-MnMEIO was found in sentinel lymph node area, showing good overlaid PET and MR image, respectively. (Reprinted with permission [17]). 


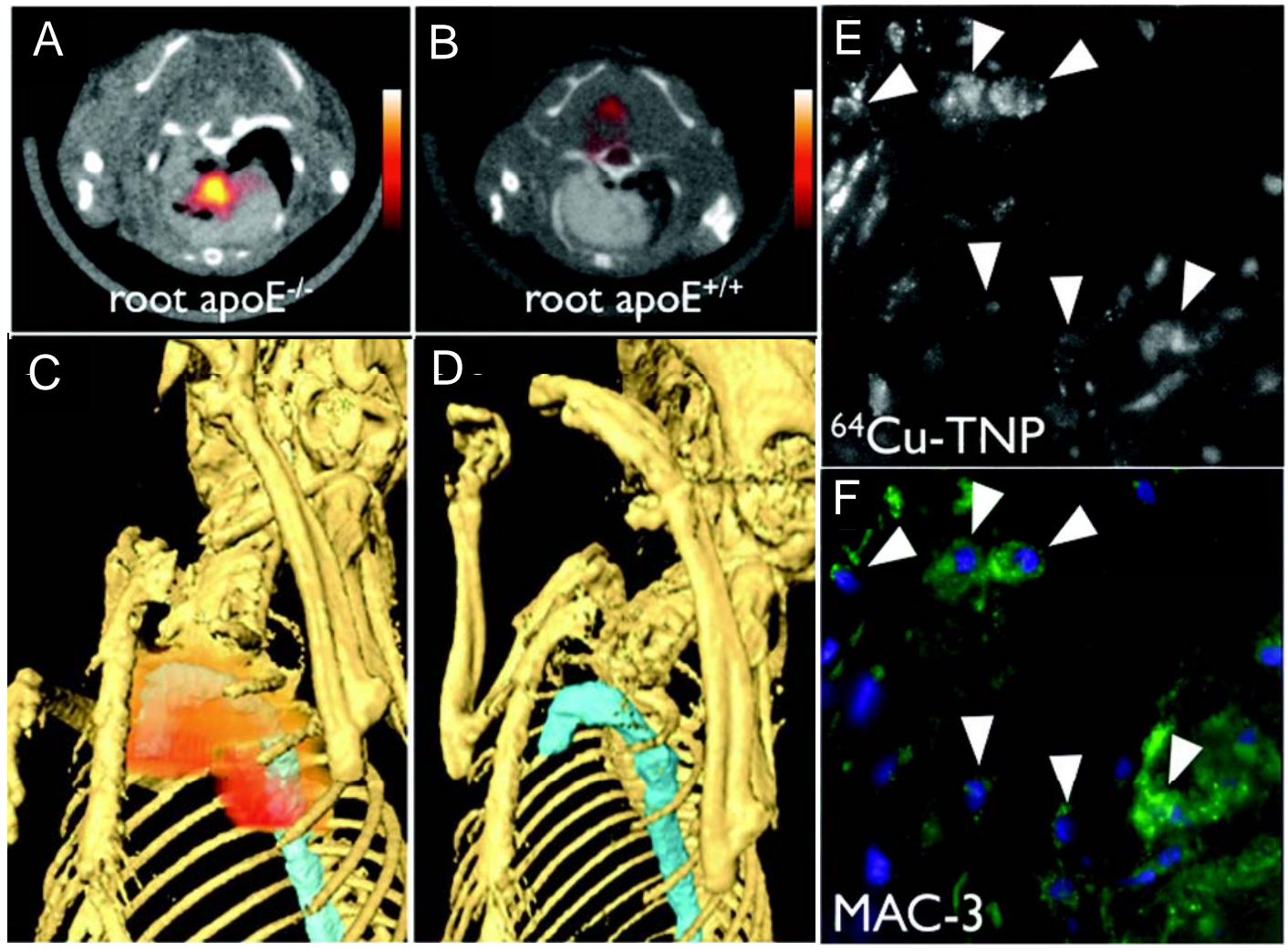

Fig. (2). Detection of atherosclerotic plaque using trimodality imaging nanoprobe in apoE-/- mice. Iron oxide magnetic core was conjugated with near-infrared fluorophore, followed by the labeling with ${ }^{64} \mathrm{Cu}$ radioisotope through DTPA chelator to yield ${ }^{64} \mathrm{Cu}$-labled tri-reporter nanoparticle $\left({ }^{64} \mathrm{Cu}\right.$-TNP). (A, B) Macrophage activity targeted by PET agents in aortic root was clearly visualized in apoE ${ }^{-/-}$mice compared to that in apoE ${ }^{+/+}$mice. Robust PET radioactivity (red) from fusion PET/CT images in proximal thoracic aorta (blue) was detected in apoE ${ }^{-/-}$ mice $(\mathbf{C})$ whereas no PET signal was observed in wild type mice $(\mathbf{D})$. (E, F) Intracellular accumulation of ${ }^{64} \mathrm{Cu}-\mathrm{TNP}$ in macrophage was found in atheromatous plaque area, showing co-localized fluorescence distribution between 64Cu-TNP and antibody for MAC-3, macrophage differentiation antigen. (Reprinted with permission [33]).

system with addition of luciferase reporter protein that enables in vivo bioluminescence image [34]. The quadruple nanoprobes possible for four different imaging were designed from original cobalt ferrite magnetic nanoparticle coated with silica shell to incorporate rhodamine fluorescence dye, which silica-based magnetic fluorescence leads to significant enhancement of fluorescence signal by caging effect as well as photostable effects against photobleaching and the biocompatibility by protection from metal cytotoxicity [35, 36]. Additionally attached with purified luciferase protein and ${ }^{68} \mathrm{Ga}$ radiotracer through $p$ SCN-bn-NOTA chelator agent, quadruple nanoplatform was created for the development of in vivo cell tracking. Bioluminescence resonance emission transfer (BRET) signal can be naturally occurred in this system due to energy transfer between luciferase and rodamine fluorescence in close proximity. Characteristics of each imaging modality including BRET effect were verified in vitro, illustrating gradual increase pattern of signal intensity in each imaging modality in dose dependent manner. Interestingly, in vivo study showed that four different modalities were clearly monitored in the same mouse, and also the reduced signal intensity of in vivo bioluminescence and fluorescence was found in intramuscular area in comparison of that in subcutaneous area whereas similar signal activity of ${ }^{68} \mathrm{Ga}$ radioisotope was detected in both thighs regardless of depth problem (Fig. 3). These results suggested that the pros and cons of characteristics of each imaging modality were experimentally well described in the same subject. This multifunctional nanoparticle probes are expected to be effective in clinical field for cancer diagnostics and therapeutics, by coupling with drug or targeting agents. However, despite these huge applications, several disadvantages in the design of multimodal imaging agents such as low synthetic efficiency of preparation process, potential interference between each imaging modality and high cost are challengeable.

\section{CONCLUSION}

With the increasing needs for improved imaging modality, hybrid PET/MR imaging systems are expected to become multifunctional diagnostic imaging probes in clinical field. This integrated PET/MR imaging approach can provide critical information by compensating for the limitation of single imaging modality in vivo with acquisition of superb resolution characteristics as well as high signal sensitivity, simultaneously. To develop a variety of combined imaging modalities, the intensive cooperation between chemical and biological knowledge should be followed for the improvement of multimodality imaging systems. Also, in order for easy approach of multimodal imaging acquisition, the development of new PET/MRI 

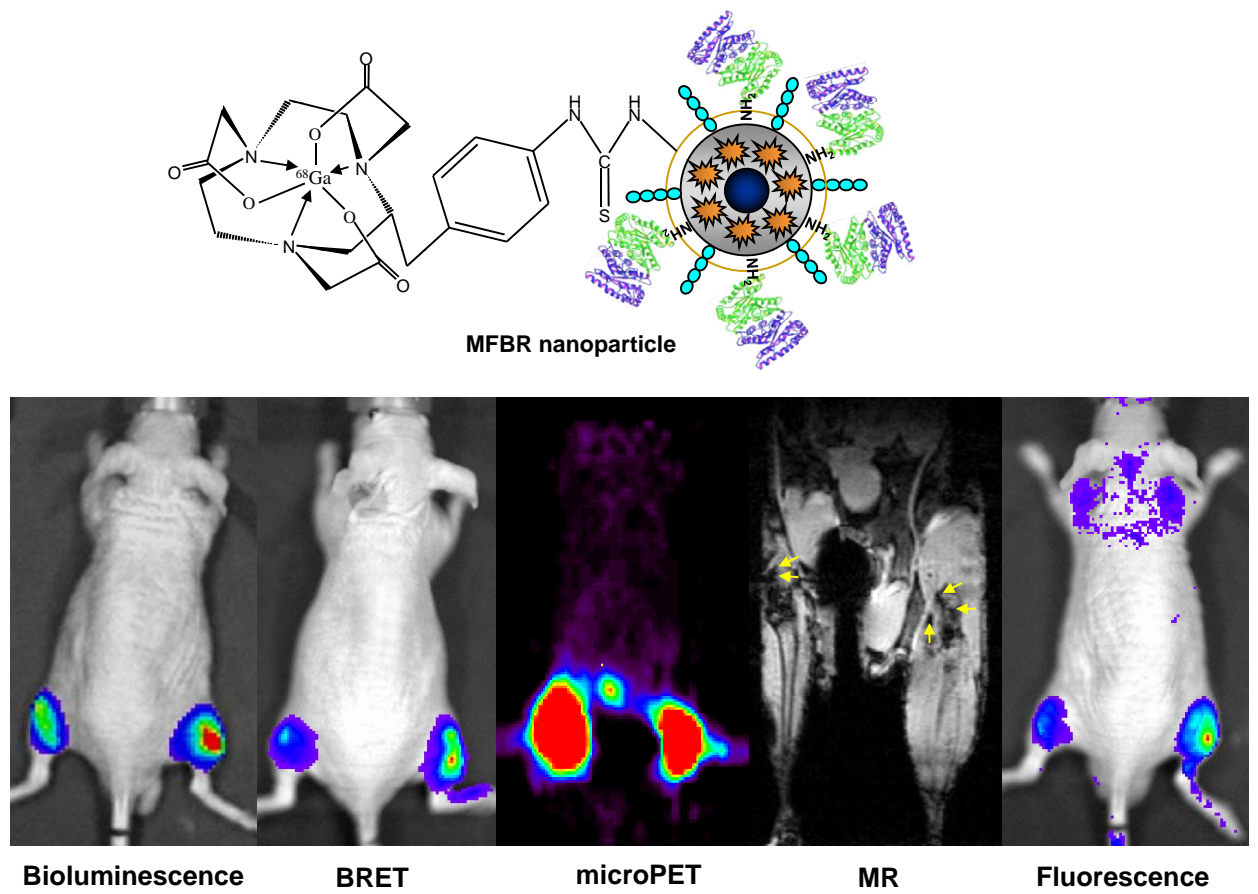

Fig. (3). Quadruple nanoparticle-based multifunctional imaging system for cell tracking in vivo. The cobalt ferrite magnetics in central core were surrounded with fluorescence dye coated with silica shell, which were conjugated with both bioluminescent luciferase protein and ${ }^{68} \mathrm{Ga}$ radioisotope to produce the MFBR (magnetic fluorescent bioluminescent radioisotope) nanoparticles. Each in vivo activity in four different imaging modalities was monitored in the same mouse. The depth independent PET and MR signals showed similar intensity in both thighs whereas the stronger bioluminescent and fluorescent signal was observed in subcutaneous region than those in intramuscular region. In vivo BRET effect between fluorescent dye and luciferase protein was also found in C6-MFBR-grafted mouse. (Reprinted with permission [34]).

fusion scanners is required. However, many challenges such as the serious interference between PET and MRI imaging devices have hindered the development of MR-compatible PET imaging scanner. Nevertheless, many trials to solve these drawbacks for PET/MR fusion scanner have been recently achieved in field of physics, by showing a variety of methodologies such as lutetium oxyorthosilicate-Avalanche photodiode (LSO-APD)-based sensor and silicon-multiplier (SiPM) [37, 38]. Based on LSO crystal scintillator, one resent report demonstrated the development of a novel multislice PET detector integrated with 7 tesla MRI scanner, showing superb PET/MR fusion images. High quality integrated PET/MR images were found in tumor and brain models through complementary multimodal imaging acquisition [39].

As rapid advances for the application of nanomaterials to biomedical research field have been progressed, the development of efficient imaging probe has been required for clinical application. A large number of nanoparticle probes have been developed as potential delivery carriers. Their characteristics such as extremely small size and numerously available functional moieties represent useful advantage for evaluation of drug distribution as well as the efficient drug delivery at the target sites. Inorganic quantum dot and iron oxide nanoparticle which possess its own potential imaging properties were widely used for biological application [40, 41]. Regarding therapeutic aspects, therapeutic efficacy of doxorubicin-loaded liposome or dextran nanoparticles was proven in many animal models $[42,43]$. Likewise, it would be possible for the developed multimodal nanoparticles to be used as therapeutic delivery agents by being encapsulated of drug such as doxorubicin in their central core or linked with cancer specific AS1411 aptamer which can block cell-proliferative signaling pathway [44].

In order for in vivo tracking of nanocarriers, the combined PET and MRI imaging systems are necessary for clinical study. In this review, we proposed the comprehensive overviews of current advances of PET/MRI-based imaging nanoprobe in vivo as well as in vitro. The developed multimodal imaging nanoprobe can present multidisplinary approach for the development of the fused PET-MRI imaging devices, drug delivery and cancer tracking. Especially, the recently developed quadruple imaging agent offers potential application, participating in studies from fluorescence imaging in vitro, to bioluminescence imaging in small animal model and finally to PET or MR imaging capable of human application. Thus, multifunctional imaging modality platform established by combination of nanotechnology, will be standing on tiptoe for development of concurrent imaging methodologies with integration of each modality [45], and will open new avenues of diagnostic and therapeutic application in biomedical imaging field.

\section{ACKNOWLEDGEMENTS}

This work was supported by the Nano Bio Regenomics Project (2005-00113) and Brain Research Center of the 21st Century Frontier Research Program funded by the Ministry of Science and Technology (2009K001257) and the WCU project of the MEST and the NRF (R31-2008-000-10103-0). 


\section{REFERENCES}

[1] Driehuys B, Nouls J, Badea A, et al. Small animal imaging with magnetic resonance microscopy. ILAR J. 2008; 49: 35-53.

[2] Lecomte R. Novel detector technology for clinical PET. Eur J Nucl Med Mol Imaging 2009; 36: S69-85.

[3] Heiss WD. The potential of PET/MR for brain imaging. Eur J Nucl Med Mol Imaging. 2009; 36: S105-12.

[4] Cherry SR. Multimodality in vivo imaging systems: twice the power or double the trouble? Ann Rev Biomed Eng 2006; 8: 35-62.

[5] Wang W, Ke S, Kwon S, et al. A new optical and nuclear duallabeled imaging agent targeting interleukin 11 receptor alpha-chain. Bioconjug Chem 2007; 18: 397-402.

[6] Ray P, De A, Min JJ, Tsien RY, Gambhir SS. Imaging tri-fusion multimodality reporter gene expression in living subjects. Cancer Res 2004; 64: 1323-30.

[7] Talanov VS, Regino CA, Kobayashi H, Bernardo M, Choyke PL, Brechbiel MW. Dendrimer-based nanoprobe for dual modality magnetic resonance and fluorescence imaging. Nano Lett 2006; 6: 1459-63.

[8] Leary SP, Liu CY, Yu C, Apuzzo ML. Toward the emergence of nanoneurosurgery: part II--nanomedicine: diagnostics and imaging at the nanoscale level. Neurosurgery 2006; 58: 805-23.

[9] Leary SP, Liu CY, Yu C, Apuzzo ML. Toward the emergence of nanoneurosurgery: part III-nanomedicine: targeted nanotherapy, nanosurgery, and progress toward the realization of nanoneurosurgery. Neurosurgery 2006; 58: 1009-26.

[10] Suri SS, Fenniri H, Singh B. Nanotechnology-based drug delivery systems. J Occup Med Toxicol 2007; 2:16.

[11] Liu Y, Miyoshi H, Nakamura M Nanomedicine for drug delivery and imaging: a promising avenue for cancer therapy and diagnosis using targeted functional nanoparticles. Int J Cancer 2007;120: 2527-37.

[12] Sharma P, Brown S, Walter G, Santra S, Moudgil B. Nanoparticles for bioimaging. Adv Colloid Interface Sci 2006; 123-126: 471-85.

[13] Schuster B. Polymeric nanoparticles as imaging probes for protein kinase activity in cells. Angew Chem Int Ed Engl 2007; 46:8744-6.

[14] Medarova Z, Pham W, Farrar C, Petkova V, Moore A. In vivo imaging of siRNA delivery and silencing in tumors. Nat Med 2007; 13:372-7.

[15] Kim KY. Nanotechnology platforms and physiological challenges for cancer therapeutics. Nanomedicine 2007; 3:103-10.

[16] Lee HY, Li Z, Chen K, et al. PET/MRI dual-modality tumor imaging using arginine-glycine-aspartic (RGD)-conjugated radiolabeled iron oxide nanoparticles. J Nucl Med 2008; 49:1371-9.

[17] Choi JS, Park JC, Nah H, et al. Hybrid nanoparticle probe for dualmodality positron emission tomography and magnetic resonance imaging. Angew Chem Int Ed Engl 2008; 47: 6259-62.

[18] Cai W, Shin DW, Chen K, et al. Peptide-labeled near-infrared quantum dots for imaging tumor vasculature in living subjects. Nano Lett 2006; 6: 669-76.

[19] Schmieder AH, Winter PM, Caruthers SD, et al. Molecular MR imaging of melanoma angiogenesis with alphanubeta3-targeted paramagnetic nanoparticles. Magn Reson Med 2005; 53: 621-7.

[20] Zhang C, Jugold M, Woenne EC, et al. Specific targeting of tumor angiogenesis by RGD-conjugated ultrasmall superparamagnetic iron oxide particles using a clinical 1.5-T magnetic resonance scanner. Cancer Res 2007; 67: 1555-62.

[21] Ellegala DB, Leong-Poi $\mathrm{H}$, Carpenter JE, et al. Imaging tumor angiogenesis with contrast ultrasound and microbubbles targeted to alpha(v)beta3. Circulation 2003; 108: 336-41.

[22] Zavaleta C, de la Zerda A, Liu Z, et al. Noninvasive Raman spectroscopy in living mice for evaluation of tumor targeting with carbon nanotubes. Nano Lett 2008; 8:2800-5.

[23] Lee JH, Huh YM, Jun YW, et al. Artificially engineered magnetic nanoparticles for ultra-sensitive molecular imaging. Nat Med 2007; 13: 95-9.
[24] Rossin R, Muro S, Welch MJ, Muzykantov VR, Schuster DP. In vivo imaging of ${ }^{64} \mathrm{Cu}$-labeled polymer nanoparticles targeted to the lung endothelium. J Nucl Med 2008; 49:103-11.

[25] Anderson SA, Glod J, Arbab AS, et al. Noninvasive MR imaging of magnetically labeled stem cells to directly identify neovasculature in a glioma model. Blood 2005; 105:420-5.

[26] Sykova E, Jendelova P. In vivo tracking of stem cells in brain and spinal cord injury. Prog Brain Res 2007; 161: 367-83.

[27] Jendelová $\mathrm{P}$, Herynek V, Urdzíková L, et al. Magnetic resonance tracking of transplanted bone marrow and embryonic stem cells labeled by iron oxide nanoparticles in rat brain and spinal cord. $\mathrm{J}$ Neurosci Res 2004; 76: 232-43.

[28] Chapon C, Jackson JS, Aboagye EO, Herlihy AH, Jones WA, Bhakoo KK. An in vivo multimodal imaging study using MRI and PET of stem cell transplantation after myocardial infarction in rats. Mol Imaging Biol 2009; 11:31-22

[29] Higuchi T, Anton M, Dumler K, et al. Combined Reporter Gene PET and Iron Oxide MRI for Monitoring Survival and Localization of Transplanted Cells in the Rat Heart. J Nucl Med 2009; 50: 10881094.

[30] Cheon J, Lee JH. Synergistically integrated nanoparticles as multimodal probes for nanobiotechnology. Acc Chem Res 2008; 41: $1630-40$

[31] Kim S, Lim YT, Soltesz EG, et al. Near-infrared fluorescent type II quantum dots for sentinel lymph node mapping. Nat Biotechnol 2004; 22: 93-7.

[32] Kobayashi H, Hama Y, Koyama Y, et al. Simultaneous multicolor imaging of five different lymphatic basins using quantum dots. Nano Lett 2007; 7: 1711-6.

[33] Nahrendorf M, Zhang H, Hembrador S, et al. Nanoparticle PETCT imaging of macrophages in inflammatory atherosclerosis. Circulation 2008; 117: 379-87.

[34] Hwang DW, Ko HY, Kim SK, Kim D, Lee DS, Kim S. Development of a quadruple imaging modality by using nanoparticles. Chemistry 2009; 15: 9387-93.

[35] Kim JS, Yoon TJ, Yu KN, et al. Toxicity and tissue distribution of magnetic nanoparticles in mice. Toxicol Sci 2006; 89: 338-47.

[36] Yoon TJ, Yu KN, Kim E, et al. Specific targeting, cell sorting, and bioimaging with smart magnetic silica core-shell nanomaterials. Small 2006; 2: 209-15.

[37] Pichler BJ, Judenhofer MS, Catana C, et al. Performance test of an LSO-APD detector in a 7-T MRI scanner for simultaneous PET/MRI. J Nucl Med 2006; 47: 639-47.

[38] Schaart DR, van Dam HT, Seifert S, et al. A novel, SiPM-arraybased, monolithic scintillator detector for PET. Phys Med Biol 2009; 54: 3501-12.

[39] Judenhofer MS, Wehrl HF, Newport DF, et al. Simultaneous PETMRI: a new approach for functional and morphological imaging. Nat Med 2008; 14: 459.

[40] Sun C, Lee JS, Zhang M. Magnetic nanoparticles in MR imaging and drug delivery. Adv Drug Deliv Rev 2008; 60: 1252-65.

[41] Michalet X, Pinaud FF, Bentolila LA, et al. Quantum dots for live cells, in vivo imaging, and diagnostics. Science 2005; 307: 538-44.

[42] Medarova Z, Rashkovetsky L, Pantazopoulos P, Moore A. Multiparametric monitoring of tumor response to chemotherapy by noninvasive imaging. Cancer Res 2009; 69: 1182-9.

[43] Krauze MT, Noble CO, Kawaguchi T, et al. Convection-enhanced delivery of nanoliposomal CPT-11 (irinotecan) and PEGylated liposomal doxorubicin (Doxil) in rodent intracranial brain tumor xenografts. Neuro Oncol 2007; 9: 393-403.

[44] Girvan AC, Teng Y, Casson LK, et al. AGRO100 inhibits activation of nuclear factor-kappaB (NF-kappaB) by forming a complex with NF-kappaB essential modulator (NEMO) and nucleolin. Mol Cancer Ther 2006; 5: 1790-9.

[45] Cai W, Chen X. Nanoplatforms for targeted molecular imaging in living subjects. Small 2007; 3: 1840-54. 